\title{
FUNCTION SPACES OF LOW BOREL COMPLEXITY
}

\author{
J. DIJKSTRA, T. GRILLIOT, D. LUTZER ${ }^{1}$ AND J. Van MILL ${ }^{2}$
}

\begin{abstract}
In this paper we investigate situations in which the space $C_{\pi}(X)$ of continuous, real-valued functions on $X$ is a Borel subset of the product space $\mathbf{R}^{X}$. We show that for completely regular, nondiscrete spaces, $C_{\pi}(X)$ cannot be a $G_{\delta}$, an $F_{\sigma}$, or a $G_{\delta \sigma}$ subset of $\mathbf{R}^{X}$, but it can be an $F_{\sigma \delta}$ or $G_{\delta \sigma \delta}$ subset.
\end{abstract}

I. Introduction Let $C_{\pi}(X)$ be the set of continuous real-valued functions on a topological space $X$, and topologize $C_{\pi}(X)$ as a subspace of the full product $\mathbf{R}^{X}$. Assuming that there are enough continuous functions on $X$ to separate points, $C_{\pi}(X)$ is a dense subspace of $\mathbf{R}^{X}$. In this paper we examine situations in which $C_{\pi}(X)$ is a Borel subset of $\mathbf{R}^{X}$ and occurs very early in the Borel hierarchy of $\mathbf{R}^{X}$. We prove that if $X$ is completely regular, $C_{\pi}(X)$ cannot be a $G_{\delta}, G_{\delta \sigma}$ or $F_{\sigma}$ subset of $\mathbf{R}^{X}$ unless $X$ is discrete. Examples show that $C_{\pi}(X)$ can be a $G_{\delta \sigma \delta}$ or $F_{\sigma \delta}$ subset of $\mathbf{R}^{X}$. In another paper [LMP] it will be shown that $C_{\pi}(X)$ can have arbitrarily high Borel or projective class in $\mathbf{R}^{X}$ and that $C_{\pi}(X)$ may be entirely outside of the projective hierarchy even when $X$ is countable.

In what follows, no separation axioms are assumed unless specifically stated, except that completely regular spaces are assumed to be $T_{1}$. However, this lack of separation is partially an illusion, since all of our main results (Theorems 1, 6, and 7) deal with situations where $C_{\pi}(X)$ is dense in $\mathbf{R}^{X}$, and that is known to be equivalent to the separation axiom "given distinct points $x$ and $y$ of $X$, some continuous $f$ : $X \rightarrow \mathbf{R}$ has $f(x) \neq f(y)$." (Such a space is said to be completely Hausdorff.) Examples 10 and 11 show the crucial role played by this separation axiom in our paper.

For any set $S$, both $\bar{S}$ and $\operatorname{cl}(S)$ will be used to denote the closure of $S$.

II. Certain discrete spaces. Our first result was originally obtained in [LM] using a complicated proof. We begin by presenting an elementary proof which has the advantage (over the proof in $[\mathbf{L M}]$ ) that no separation axioms are assumed.

1. Theorem. For any space $X$, if $C_{\pi}(X)$ contains a dense $G_{\delta}$ subset of $\mathbf{R}^{X}$ then $X$ is discrete.

Proof. Suppose $X$ is not discrete. Then there is a function $g \in \mathbf{R}^{X}-C_{\pi}(X)$. Define $\Phi: \mathbf{R}^{X} \rightarrow \mathbf{R}^{X}$ by $\Phi(f)=f+g$. Then $\Phi$ is a homeomorphism of $\mathbf{R}^{X}$ onto itself

Received by the editors February 2, 1984 and, in revised form, May 11, 1984.

1980 Mathematics Subject Classification. Primary 54C35, 54H05, 54C50.

${ }^{1}$ Partially supported by NSF grant MCS80-016617.

${ }^{2}$ Partially supported by NATO grant 1927 . 
and $\Phi\left[C_{\pi}(X)\right] \subset \mathbf{R}^{X}-C_{\pi}(X)$. Thus $\mathbf{R}^{X}-C_{\pi}(X)$ also contains a dense $G_{\delta}$ subset of $\mathbf{R}^{X}$, which is impossible because $\mathbf{R}^{X}$ is a Baire space [dG] and in a Baire space any pair of dense $G_{\delta}$ sets has nonvoid intersection.

We next examine the situation where $C_{\pi}(X)$ is a $G_{\delta \sigma}$ subset of $\mathbf{R}^{X}$. For completely Hausdorff spaces this also occurs only when $X$ is discrete, but the proof is surprisingly complicated compared to the proof of Theorem 1.

2. Proposition. The following properties of a completely regular space $X$ are equivalent:

(a) given any collection $\left\{\left(D_{\alpha}, C_{\alpha}\right) \mid \alpha \in A\right\}$ where $D_{\alpha} \subset C_{\alpha}, D_{\alpha}$ is a zero set, $C_{\alpha}$ is a cozero set and $C_{\alpha} \cap C_{\beta}=\varnothing$ whenever $\alpha \neq \beta$, the set $\bigcup\left\{D_{\alpha} \mid \alpha \in A\right\}$ is a zero set;

(b) the union of any disjoint collection of zero sets is again a zero set;

(c) $X$ is discrete.

Proof. Obviously (c) $\Rightarrow(\mathrm{b}) \Rightarrow(\mathrm{a})$. To prove the converses, we need to know that (a) implies

(*) Each zero set in $X$ is a cozero set in $X$.

Assume (a) holds and let $C$ be a cozero set in $X$. Then there is a continuous $f$ : $X \rightarrow[0,1]$ having $C=f^{-1}[(0,1]]$. Define subsets $E_{n}$ and $E_{n}^{\prime}$ of $(0,1]$ as follows:

$$
E_{n}=\left[2^{-(2 n-1)}, 2^{-(2 n-2)}\right], \quad E_{n}^{\prime}=\left[2^{-2 n}, 2^{-(2 n-1)}\right] .
$$

Then

$$
(0,1]=\bigcup\left\{E_{n} \cup E_{n}^{\prime} \mid n \geqslant 1\right\} .
$$

Let $D_{n}=f^{-1}\left[E_{n}\right]$ and $C_{n}=f^{-1}\left[\left(3 / 2^{2 n+1}, 3 / 2^{2 n-1}\right)\right]$. Then (a) may be applied to the collection $\left\{\left(D_{n}, C_{n}\right) \mid n \geqslant 1\right\}$ to show that $S=\bigcup\left\{f^{-1}\left[E_{n}\right] \mid n \geqslant 1\right\}$ is a zero set. Analogously, $T=\bigcup\left\{f^{-1}\left[E_{n}^{\prime}\right] \mid n \geqslant 1\right\}$ is also a zero set. But then so is $S \cup T=$ $f^{-1}[(0,1]]$, i.e., each cozero subset of $X$ is a zero set. Assertion $(*)$ follows by taking complements.

To prove (b), given any disjoint family $\left\{D_{\alpha} \mid \alpha \in A\right\}$ of zero sets, we let $C_{\alpha}=D_{\alpha}$. By (*), each $C_{\alpha}$ is a cozero set so that we may apply (a) to the collection $\left\{\left(D_{\alpha}, C_{\alpha}\right) \mid \alpha \in A\right\}$ to conclude that $\bigcup\left\{D_{\alpha} \mid \alpha \in A\right\}$ is a zero set, as required.

Finally, assume (b) and let $p \in X$. Suppose $\{p\}$ is not open. Let $\mathscr{D}$ be a maximal disjoint collection of zero sets, each contained in $X-\{p\}$. Let $W$ be any neighborhood of $p$. Because $\{p\} \neq W$, if $W \cap \cup \mathscr{D}=\varnothing$, then we could find a zero set $Z \subset W-\{p\}$ contrary to maximality of $\mathscr{D}$. Hence $\cup \mathscr{D}$ is dense in $X$. But, from (b), $\cup \mathscr{D}$ is a zero set so $\cup \mathscr{D}$ is both closed and dense in $X$ contrary to $\cup \mathscr{D} \subset X-\{p\}$. Hence $X$ must be discrete.

3. REMARK. In general, assertion (*) in the proof of Lemma 2 is not equivalent to discreteness of $X$ : consider $X=\left[0, \omega_{1}\right]$, in which each countable ordinal is isolated, while $\omega_{1}$ has its usual neighborhoods. However, if each point of $X$ is a limit point of a countable set, or if each point of $X$ is a $G_{\delta}$ in $X$, then (*) does imply that $X$ is discrete. 
In order to apply Proposition 2 to the case where $C_{\pi}(X)$ is a $G_{\delta \sigma}$ subset of $\mathbf{R}^{X}$, we need two rather technical lemmas. In what follows, given $f \in \mathbf{R}^{X}$ and $B \subset X$,

$$
E(f, B)=\left\{g \in \mathbf{R}^{X} \mid g(x)=f(x) \text { for each } x \in B\right\} .
$$

4. Lemma. Let $A$ be any set and let $H$ be a $G_{\delta}$-subset of $\mathbf{R}^{A}$. Suppose $B_{0}$ is a fixed finite subset of $A$. Let $e: B_{0} \rightarrow \mathbf{R}$ be a fixed function and suppose $\delta: A \rightarrow[0, \infty)$ is given. Let

$$
P=\left\{g \in \mathbf{R}^{A} \mid 0 \leqslant g(x) \leqslant \delta(x) \text { for } x \in A-B_{0}\right\} \cap E\left(e, B_{0}\right) .
$$

Then either

(a) there is a finite set $B \subset A-B_{0}$ and a function $h \in P$ having $P \cap E(h, B) \cap H$ $=\varnothing ;$ or

(b) there are functions $g_{0}, g_{1} \in H$ having $g_{0}(x)+g_{1}(x)=\delta(x)$ for each $x \in A-$ $B_{0}$.

Proof. Write $H=\bigcap\left\{U_{n} \mid n \geqslant 1\right\}$ where $U_{1} \supset U_{2} \supset \cdots$ are open in $\mathbf{R}^{A}$. Suppose (a) fails. Then for each $h \in P$ and each finite $B \subset A-B_{0}, P \cap E(h, B) \cap H \neq \varnothing$.

Fix $f_{1} \in P$ and let $B=\varnothing$. There is a function $\hat{f}_{1} \in P \cap H \cap E\left(f_{1}, \varnothing\right)$. Because $\hat{f}_{1} \in H \subset U_{1}$, there is a finite set $B_{1} \supset B_{0}$ such that $E\left(\hat{f}_{1}, B_{1}\right) \subset U_{1}$.

Suppose $n \geqslant 1$ and we have constructed finite sets $B_{1}, \ldots, B_{n}$ and functions $f_{k}, \hat{f}_{k}$ for $1 \leqslant k \leqslant n$ satisfying:

(a) $B_{0} \subset B_{1} \subset \cdots \subset B_{n}$,

(b) if $k+1 \leqslant n$ then

$$
f_{k+1}= \begin{cases}e(x) & \text { if } x \in B_{0}, \\ \delta(x)-\hat{f}_{k}(x) & \text { if } x \in B_{k}-B_{0}, \\ 0 & \text { otherwise }\end{cases}
$$

(c) $f_{k}, \hat{f}_{k} \in P$ for $1 \leqslant k \leqslant n$,

(d) $\hat{f}_{k+1} \in P \cap H \cap E\left(f_{k+1}, B_{k}\right)$ if $k+1 \leqslant n$,

(e) $E\left(\hat{f}_{k}, B_{k}\right) \subset U_{k}$ if $k \leqslant n$.

We obtain $f_{n+1}, \hat{f}_{n+1}$ and $B_{n+1}$ as follows. Define

$$
f_{n+1}(x)= \begin{cases}e(x) & \text { if } x \in B_{0} \\ \delta(x)-\hat{f}_{n}(x) & \text { if } x \in B_{n}-B_{0} \\ 0 & \text { otherwise. }\end{cases}
$$

Because $\hat{f}_{n} \in P$, we know that $0 \leqslant \hat{f}_{n}(x) \leqslant \delta(x)$ for each $x \in A-B_{0}$. Hence $0 \leqslant \delta(x)-\hat{f}_{n}(x) \leqslant \delta(x)$ for each $x \in B_{n}-B_{0}$, so that $f_{n+1} \in P$. Since (a) fails, $P \cap H \cap E\left(f_{n+1}, B_{n}\right) \neq \varnothing$. Choose $\hat{f}_{n+1} \in P \cap H \cap E\left(f_{n+1}, B_{n}\right)$. Since $\hat{f}_{n+1} \in H$ $\subset U_{n+1}$, there is a finite set $B_{n+1} \supset B_{n}$ such that $E\left(\hat{f}_{n+1}, B_{n+1}\right) \subset U_{n+1}$. Therefore $f_{n+1}, \hat{f}_{n+1}$ and $B_{n+1}$ are defined, as required.

Fix $n$ and $x \in B_{n}-B_{0}$. Then $\hat{f}_{n+1}(x)=f_{n+1}(x)=\delta(x)-\hat{f}_{n}(x)$, and $\hat{f}_{n+2}(x)=$ $f_{n+2}(x)=\delta(x)-\hat{f}_{n+1}(x)$. Therefore, $\hat{f}_{n+2}(x)=\delta(x)-\left(\delta(x)-\hat{f}_{n}(x)\right)=\hat{f}_{n}(x)$. It follows that for $x \in B_{2 n}, \hat{f}_{2 k}(x)=\hat{f}_{2 n}(x)$ for every $k \geqslant n$. Therefore we may define a function

$$
g_{0}(x)= \begin{cases}\hat{f}_{2 n}(x) & \text { if } x \in B_{2 n} \text { for some } n \\ 0 & \text { if } x \in A-\bigcup_{n=1}^{\infty} B_{2 n}=A-\bigcup_{n=0}^{\infty} B_{n} .\end{cases}
$$


Fix $n$ and $x \in B_{2 n}$. Since $g_{0}(x)=\hat{f}_{2 n}(x)$ we see that $g_{0} \in E\left(\hat{f}_{2 n}, B_{2 n}\right) \subset U_{2 n}$. Since $U_{1} \supset U_{2} \supset \cdots$ we have $g_{0} \in \bigcap_{n=1}^{\infty} U_{n}=H$. Now define

$$
g_{1}(x)= \begin{cases}\delta(x)-g_{0}(x) & \text { if } x \in A-B_{0}, \\ e(x) & \text { if } x \in B_{0} .\end{cases}
$$

Fix $n$ and $x \in B_{2 n+1}$. If $x \in B_{0}$ then $g_{1}(x)=e(x)=\hat{f}_{2 n+1}(x)$, so suppose $x \in$ $B_{2 n+1}-B_{0}$. Then $x \in B_{2 n+2}$, so that $g_{0}(x)=\hat{f}_{2 n+2}(x)$ and $\hat{f}_{2 n+2}(x)=\delta(x)-$ $\hat{f}_{2 n+1}(x)$, because $x \in B_{2 n+1}-B_{0}$. Thus $g_{0}(x)=\delta(x)-\hat{f}_{2 n+1}(x)$ for each $x \in$ $B_{2 n+1}-B_{0}$, and so $\hat{f}_{2 n+1}(x)=\delta(x)-g_{0}(x)=g_{1}(x)$. Therefore $g_{1} \in$ $E\left(\hat{f}_{2 n+1}, B_{2 n+1}\right) \subset U_{2 n+1}$. Hence $g_{1} \in \bigcap_{1}^{\infty} U_{n}=H$. By definition of $g_{1}$, if $x \in A-$ $B_{0}, g_{0}(x)+g_{1}(x)=\delta(x)$, as required.

5. Lemma. Let $A$ be any set, and let $G$ be a $G_{\delta \sigma}$ subset of $\mathbf{R}^{A}$ which is an additive subgroup of $\mathbf{R}^{A}$. Suppose $G$ contains every function $w \in \mathbf{R}^{A}$ such that $\{x \in A|| w(x) \mid$ $\geqslant \varepsilon\}$ is finite for each $\varepsilon>0$. Then each bounded function from $A$ to $\mathbf{R}$ belongs to $G$.

Proof. Since each bounded function from $A$ to $\mathbf{R}$ is the difference of two nonnegative bounded functions, it will be enough to show that each bounded $f$ : $A \rightarrow[0, \infty)$ belongs to $G$. Suppose $f: A \rightarrow[0, \infty)$ is bounded and does not belong to $G$. Write $G=\cup_{1}^{\infty} H_{n}$ where $H_{1} \subset H_{2} \subset \cdots$ and each $H_{n}$ is a $G_{\delta}$ subset of $\mathbf{R}^{A}$.

Let $P_{1}=\left\{g \in \mathbf{R}^{A} \mid 0 \leqslant g(x) \leqslant f(x)\right.$ for all $\left.x \in A\right\}$. We apply Lemma 4 to $P_{1}$ and $H_{1}$. If there are functions $g_{0}, g_{1} \in H_{1}$ with $g_{0}+g_{1}=f$, it would follow that $g_{0}+g_{1}=f$ is also in $G$. Hence there is a finite set $A_{1} \subset A$ and a function $h_{1} \in P_{1}$ such that $P_{1} \cap H_{1} \cap E\left(h_{1}, A_{1}\right)=\varnothing$.

For induction hypothesis suppose $n \geqslant 1$ and that we have pairwise disjoint finite sets $A_{1}, \ldots, A_{n}$ and functions $h_{1}, \ldots, h_{n}$ satisfying:

(a) $\left.h_{k+1}\right|_{B}=\left.h_{k}\right|_{B}$ provided $k+1 \leqslant n$ where $B=A_{1} \cup \cdots \cup A_{k}$;

(b) $h_{k} \in P_{k}=\left\{g \in \mathbf{R}^{A} \mid 0 \leqslant g(x) \leqslant(1 / k) f(x)\right.$ for $x \in A-\left(A_{1} \cup \cdots \cup\right.$ $\left.\left.A_{k-1}\right)\right\} \cap\left(\cap_{j=1}^{k-1} E\left(h_{j}, A_{j}\right)\right)$ provided $k \leqslant n$;

(c) $H_{k} \cap P_{k} \cap E\left(h_{k}, A_{k}\right)=\varnothing$ provided $k \leqslant n$.

To obtain $h_{n+1}$ and $A_{n+1}$ we proceed as follows. Let $B_{0}=A_{1} \cup \cdots \cup A_{n}$ and define $e: B_{0} \rightarrow[0, \infty)$ by $e(x)=h_{k}(x)$ when $x \in A_{k}$. Let

$$
P_{n+1}=\left\{g \in \mathbf{R}^{A} \mid 0 \leqslant g(x) \leqslant(n+1)^{-1} f(x) \text { for } x \in A-B_{0}\right\} \cap E\left(e, B_{0}\right) .
$$

Apply Lemma 4 to $P_{n+1}$ and $H_{n+1}$. If there are functions $g_{0}, g_{1} \in H_{n+1} \subset G$ having $g_{0}(x)+g_{1}(x)=(n+1)^{-1} f(x)$ for each $x \in A-B_{0}$, define

$$
d(x)=\left\{\begin{array}{l}
f(x)-(n+1)\left(g_{0}(x)+g_{1}(x)\right) \text { for } x \in B_{0}, \\
0 \text { if } x \in A-B_{0}
\end{array}\right.
$$

Since $\{x \in A \mid d(x) \neq 0\}$ is finite, $d \in G$. Therefore $d+(n+1)\left(g_{0}+g_{1}\right) \in G$. But for each $x \in A, d(x)+(n+1)\left(g_{0}(x)+g_{1}(x)\right)=f(x)$, contradicting $f \notin G$. Hence there is a function $h_{n+1} \in P_{n+1}$ and a finite set $A_{n+1} \subset A-B_{0}$ such that $\varnothing=H_{n+1}$ $\cap P_{n+1} \cap E\left(h_{n+1}, A_{n+1}\right)$. Since $h_{n+1} \in P_{n+1} \subset E\left(e, B_{0}\right)$, we have $\left.h_{n+1}\right|_{A_{k}}=\left.h_{k}\right|_{A_{k}}$ whenever $k \leqslant n$. Thus the induction continues. 
The induction generates pairwise disjoint finite sets $A_{1}, A_{2}, \ldots$ and functions $h_{k}$ such that if $m<n$ then $\left.h_{n}\right|_{A_{m}}=\left.h_{m}\right|_{A_{m}}$. Therefore the function

$$
h(x)= \begin{cases}h_{k}(x) & \text { if } x \in A_{k} \text { for some } k, \\ 0 & \text { if } x \in A-\bigcup\left\{A_{k} \mid k \geqslant 1\right\}\end{cases}
$$

is well defined. We show that $h \in G$. Fix a number $M>0$ with $|f(x)| \leqslant M$ for each $x$. Fix $\varepsilon>0$ and $n$ so large that $M / n<\varepsilon$. Suppose $x \in A_{k}$ for some $k>n$. Then $h(x)=h_{k}(x)$. Since $h_{k}$ belongs to $\left\{g \in \mathbf{R}^{A} \mid 0 \leqslant g(y) \leqslant(1 / k) f(y)\right.$ for $y \in A-$ $\left.\left(A_{1} \cup \cdots \cup A_{k-1}\right)\right\}$, and since $A_{k} \cap\left(A_{1} \cup \cdots \cup A_{k-1}\right)=\varnothing$, we have $0 \leqslant h_{k}(x)$ $\leqslant(1 / k) f(x) \leqslant(1 / k) M<\varepsilon$. Therefore $\{x \in A|| h(x) \mid \geqslant \varepsilon\} \subset A_{1} \cup \cdots \cup A_{n}$, which is finite. It follows that $h \in G$. But then $h \in H_{n}$ for some $n$, so that $h \in H_{n} \cap P_{n} \cap E\left(h_{n}, A_{n}\right)$, which is impossible by induction hypothesis. That contradiction completes the proof.

We are now prepared to prove our main theorem.

6. Theorem. For any space $X$, if $C_{\pi}(X)$ is a dense $G_{\delta \sigma}$ subset of $\mathbf{R}^{X}$, then $X$ is discrete.

Proof. Suppose Theorem 6 has been proved for all completely regular spaces, and suppose that $\mathscr{T}$ is a topology on $X$ such that $C_{\pi}(X, \mathscr{T})$ is a dense $G_{\delta \sigma}$ subset of $\mathbf{R}^{X}$. Then $(X, \mathscr{T})$ is completely Hausdorff (cf. $\S 1)$. Let $\mathscr{S}$ be the topology on $X$ having the family $\left\{f^{-1}[U] \mid f \in C_{\pi}(X, \mathscr{T})\right.$ and $U$ is open in $\left.\mathbf{R}\right\}$ as a subbase. Then $\mathscr{S} \subset \mathscr{T}$ and $(X, \mathscr{S})$ is completely regular and Hausdorff. Furthermore $C_{\pi}(X, \mathscr{T})$ and $C_{\pi}(X, \mathscr{S})$ are identical subsets of $\mathbf{R}^{X}$ so that $C_{\pi}(X, \mathscr{S})$ is a dense $G_{\delta \sigma}$ subset of $\mathbf{R}^{X}$. But then $(X, \mathscr{S})$ is a discrete space. Because $\mathscr{S} \subset \mathscr{T}$, so is $(X, \mathscr{T})$. Therefore it will be enough to prove Theorem 6 for an arbitrary completely regular space $X$.

According to Proposition 2, it will be enough to show that $\bigcup\left\{D_{\alpha}: \alpha \in A\right\}$ is a zero set provided $D_{\alpha}$ is a zero set contained in a cozero set $C_{\alpha}$, where $\left\{C_{\alpha} \mid \alpha \in A\right\}$ is a disjoint family. Choose a continuous function $f_{\alpha}: X \rightarrow[0,1]$ having $f_{\alpha}^{-1}[\{1\}]=D_{\alpha}$ and $\operatorname{cl}_{X}\left(f_{\alpha}^{-1}[(0,1]]\right) \subset C_{\alpha}$. Define $h(x)=\sum\left\{f_{\alpha}(x) \mid \alpha \in A\right\}$. Since the sets $C_{\alpha}$ are pairwise disjoint, $h$ is well defined and $h[X] \subset[0,1]$. Since $h^{-1}[\{1\}]=\bigcup\left\{D_{\alpha} \mid \alpha \in A\right\}$, it will be enough to prove that $h$ is continuous.

Define a function $\Phi: \mathbf{R}^{A} \rightarrow \mathbf{R}^{X}$ by the rule that if $u \in \mathbf{R}^{A}$ then $\Phi(u)=\sum\left\{u(\alpha) f_{\alpha}\right.$ : $\alpha \in A\}$. (Because $\left\{C_{\alpha} \mid \alpha \in A\right\}$ is a disjoint collection, $\Phi(u) \in \mathbf{R}^{X}$.) Clearly $\Phi$ is one-to-one. In order to show that $\Phi$ is continuous, fix a finite set $F \subset X$ and a basic neighborhood $N(\Phi(u), F, \varepsilon)=\left\{g \in \mathbf{R}^{X}|| \Phi(u)(x)-g(x) \mid<\varepsilon\right.$ for each $x$ in $\left.F\right\}$ of $\Phi(u)$ in $\mathbf{R}^{X}$. Let $A_{0}=\left\{\alpha \in A \mid C_{\alpha} \cap F \neq \varnothing\right\}$. Then $A_{0}$ is finite and the set

$$
M\left(u, A_{0}, \varepsilon\right)=\left\{v \in \mathbf{R}^{A}|| u(\alpha)-v(\alpha) \mid<\varepsilon \text { for each } \alpha \in A_{0}\right\}
$$

is a basic neighborhood of $u$ in $\mathbf{R}^{A}$. Fix $v \in M\left(u, A_{0}, \varepsilon\right)$ and consider $\mid \Phi(u)(x)-$ $\Phi(v)(x) \mid$, where $x \in F$. If $x \notin \bigcup\left\{C_{\alpha} \mid \alpha \in A\right\}$, then $f_{\alpha}(x)=0$ for each $\alpha \in A$, so that $\Phi(u)(x)=0=\Phi(v)(x)$. If $x \in C_{\beta}$ for some $\beta \in A$, then $\beta \in A_{0}$ so that

$$
|\Phi(u)(x)-\Phi(v)(x)|=\left|u(\beta) f_{\beta}(x)-v(\beta) f_{\beta}(x)\right| \leqslant|u(\beta)-v(\beta)|<\varepsilon
$$

because $f_{\beta}[X] \subset[0,1]$. Hence, $\Phi\left[M\left(u, A_{0}, \varepsilon\right)\right] \subset N(\phi(u), F, \varepsilon)$, as required. 
Since $C_{\pi}(X)$ is a $G_{\delta \sigma}$ subset of $\mathbf{R}^{X}$, it follows that the set $H=\Phi^{-1}\left[C_{\pi}(X)\right]$ is a $G_{\delta \sigma}$ subset of $\mathbf{R}^{A}$. Obviously, $H$ is an additive subgroup of $\mathbf{R}^{A}$. Suppose $u \in \mathbf{R}^{A}$ has the property that for each $\varepsilon>0$ the set $\{\alpha \in A|| u(\alpha) \mid \geqslant \varepsilon\}$ is finite. Consider the function $g=\Phi(u) \in \mathbf{R}^{X}$. To show that $g \in C_{\pi}(X)$, fix $x \in X$. If $x \in \bigcup\left\{C_{\alpha} \mid \alpha \in A\right\}$, there is a unique $\alpha$ with $x \in C_{\alpha}$. Since $\left.g\right|_{C_{\alpha}}=\left.u(\alpha) f_{\alpha}\right|_{C_{\alpha}}$ and $C_{\alpha}$ is a neighborhood of $x, g$ is continuous at $x$. Hence, suppose $x \notin \bigcup\left\{C_{\alpha} \mid \alpha \in A\right\}$. Then $g(x)=0$. Fix $\varepsilon>0$ and consider the set $S=g^{-1}[(-\varepsilon, \varepsilon)]$. By assumption, $B=\left\{\alpha \in A|| u_{\alpha} \mid \geqslant \varepsilon\right\}$ is finite, say $B=\left\{\alpha_{1}, \ldots, \alpha_{n}\right\}$. Let

$$
W=X-\cup\left\{\operatorname{cl} f_{\alpha_{i}}^{-i}[(0,1]] \mid 1 \leqslant i \leqslant n\right\} .
$$

Then $W$ is open and, since $\operatorname{cl} f_{\alpha_{i}}^{-1}[(0,1]] \subset C_{\alpha_{i}}, x \in W$. Let $z \in W$. If $z \notin$ $\bigcup\left\{C_{\alpha} \mid \alpha \in A\right\}$, then $g(z)=0$, so $z \in S$. If $z \in \bigcup\left\{C_{\alpha} \mid \alpha \in A\right\}$, there is a unique $\beta \in A$ having $z \in C_{\beta}$ and then $g(z)=u(\beta) f_{\beta}(z)$. There are two cases. If $\beta \in B$, then $z \in C_{\beta}-\operatorname{cl}\left[f_{\beta}^{-1}[(0,1]]\right]$ so that $f_{\beta}(z)=0$. Then $g(z)=0$ and so $z \in S$. Finally, if $\beta \notin B$ then $|u(\beta)|<\varepsilon$ so that $\left|u(\beta) f_{\beta}(z)\right| \leqslant|u(\beta)|<\varepsilon$, because $f_{\beta}[X] \subset[0,1]$, so that $z \in S$. Therefore $W$ is a neighborhood of $x$ which is contained in $S=g^{-1}[(-\varepsilon, \varepsilon)]$. Hence $g$ is continuous. Because $g$ is continuous, $u \in \Phi^{-1}\left[C_{\pi}(X)\right]=H$. Now apply Lemma 5 to $H$, concluding that the constant function 1 belongs to $H$. But then $\Phi(1) \in C_{\pi}(X)$, i.e., the function $\sum\left\{f_{\alpha} \mid \alpha \in A\right\}$ is continuous, as required to prove Theorem 6.

If $X$ happens to be countable, then $\mathbf{R}^{X}$ is metrizable, so that each $F_{\sigma}$ set is a $G_{\delta \sigma}$ set, showing that $C_{\pi}(X)$ is not an $F_{\sigma}$ unless $X$ is discrete. Different techniques show that the same conclusion holds even if $X$ is not countable.

7. TheOREM. For any space $X$, if $C_{\pi}(X)$ is a dense $F_{\sigma}$ subspace of $\mathbf{R}^{X}$ then $X$ is discrete.

Proof. As in the proof of Theorem 6, it will be enough to prove this result for an arbitrary completely regular space $X$.

Assume that $X$ has a nonisolated point $p$ and that $C_{\pi}(X)=\cup_{i=0}^{\infty} F_{i}$, where $F_{0}=\varnothing$ and every $F_{i}$ is closed in $\mathbf{R}^{X}$. We shall construct inductively a monotone sequence $f_{0} \leqslant f_{1} \leqslant f_{2} \leqslant \cdots$ in $[0,1]^{X}$ and subsets $U_{0} \supset \bar{U}_{1} \supset U_{1} \supset \bar{U}_{2} \supset U_{2} \supset \cdots$ of $X$ with the properties:

(a) $U_{i}$ is an open neighborhood of $p$ in $X$,

(b) $f_{i}(p)=1$,

(c) $f_{i}\left(U_{i}-\{p\}\right)=\left\{1-2^{-i}\right\}$,

(d) $\left.f_{i}\right|_{X-\{p\}}$ is continuous,

(e) $\left.f_{i+1}\right|_{X-U_{i}}=\left.f_{i}\right|_{X-U_{i}}$, and

(f) every $f \in \mathbf{R}^{X}$ that coincides with $f_{i}$ on $\left(X-U_{i}\right) \cup\{p\}$ is not an element of $F_{i}$.

Assume now that we have proved the existence of these sequences. Obviously, the pointwise limit of $\left(f_{i}\right)_{i=1}^{\infty}$ exists. Call this limit $f$ and note that $\left.f\right|_{X-U_{i}}=\left.f_{i}\right|_{X-U_{i}}$ for every $i$ and that $f\left(\cap_{i=0}^{\infty} U_{i}\right)=\{1\}$. This means, according to the induction hypothesis, that $f \notin \bigcup_{i=0}^{\infty} F_{i}$. We shall see, however, that $f$ is continuous. If $x \notin \bigcap_{i=0}^{\infty} U_{i}$, then for some $i, x \notin \bar{U}_{i}$ and $\left.f\right|_{X-\bar{U}_{i}}=\left.f_{i}\right|_{X-\bar{U}_{i}}$. Since $\left.f_{i}\right|_{X-\{p\}}$ is continuous, $f$ is continuous at $x$. If $x \in \bigcap_{i=0}^{\infty} U_{i}$, then $U_{i}$ is a neighborhood of $x$ such that $f_{i}\left(U_{i}\right) \subset\left\{1-2^{-i}, 1\right\}$. 
Consequently, $f_{j}\left(U_{i}\right) \subset\left[1-2^{-i}, 1\right]$ for every $j \geqslant i$ and hence $f\left(U_{i}\right) \subset\left[1-2^{-i}, 1\right]$. This yields the continuity of $f$ at $x$. Hence we have $f \in C_{\pi}(X)$, which contradicts $f \notin \bigcup_{i=0}^{\infty} F_{i}$.

It remains to perform the induction. As basis step we put $f_{0}(p)=1, f_{0}(x)=0$ if $x \neq p$, and $U_{0}=X$. Assume now that $f_{i}$ and $U_{i}$ have been determined. Let $\phi$ be a continuous function from $X$ into $\left[0,2^{-i}\right]$ such that $\phi\left(X-U_{i}\right) \subset\{0\}$ and $\phi(p)=2^{-i}$. Define $f_{i+1}: X \rightarrow[0,1]$ by $f_{i+1}(p)=1$ and

$$
f_{i+1}(x)=f_{i}(x)+\min \left\{2^{-i-1}, \phi(x)\right\} \quad \text { if } x \neq p .
$$

Note that $V=\phi^{-1}\left(\left(2^{-i-1}, 2^{-i}\right]\right)$ is a neighborhood of $p$ with $f_{i+1}(V-\{p\})=\{1-$ $\left.2^{-i-1}\right\}$. Since $p$ is not isolated, this means that $f_{i+1}$ is not continuous and hence not an element of $F_{i+1}$. Because $\mathbf{R}^{X}$ carries the product topology, there exists a finite set $A \subset X$ such that every $f \in \mathbf{R}^{X}$ that coincides with $f_{i+1}$ on $A$ is not in $F_{i+1}$. Put $U_{i+1}=(V-A) \cup\{p\}$. One easily verifies that the pair $\left(f_{i+1}, U_{i+1}\right)$ satisfies the induction hypothesis.

III. Examples. We begin by showing that the lowest Borel class to which $C_{\pi}(X)$ can belong for a nondiscrete completely regular space $X$ is $F_{\sigma \delta} \cap G_{\delta \sigma \delta}$.

8. Proposition. If $X$ is any countable metric space, then $C_{\pi}(X)$ is an $F_{\sigma \delta}$ subset of $\mathbf{R}^{X}$ (and hence $a G_{\delta \sigma \delta}$ subset, too).

Proof. Let $\rho$ be a metric on $X$, and for $x \in X$ and $\varepsilon>0$ let $B(x, \varepsilon)=$ $\{y \in X \mid \rho(x, y)<\varepsilon\}$. Then the $\varepsilon-\delta$ definition of continuity shows that $C_{\pi}(X)$ is the set

$$
\bigcap_{x \in X} \bigcap_{n=1}^{\infty} \bigcup_{m=1}^{\infty}\left\{g \in \mathbf{R}^{X} \mid g[B(x, 1 / m)] \subset[g(x)-1 / n, g(x)+1 / n]\right\} .
$$

Since each set $\left\{g \in \mathbf{R}^{X} \mid g[B(x, 1 / m)] \subset[g(x)-1 / n, g(x)+1 / n]\right\}$ is a closed subset of $\mathbf{R}^{X}, C_{\pi}(X)$ is an $F_{\sigma \delta}$ subset in $\mathbf{R}^{X}$, as claimed.

Our next example shows that Proposition 8 does not characterize metrizability in countable spaces:

9. EXAMPLE. There is a countable, regular, nonmetrizable space $X$ for which $C_{\pi}(X)$ is an $F_{\sigma \delta}$ subset of $\mathbf{R}^{X}$.

Proof. Let $X_{k}=\{(1 / n, 1 / n k) \mid 1 \leqslant n<\infty\} \cup\{(0,0)\}$ for $k \geqslant 1$, and let $X=$ $\cup\left\{X_{k} \mid 1 \leqslant k<\infty\right\}$. Each $X_{k}$ is topologized in such a way that $X_{k}$ becomes a convergent sequence with limit $(0,0)$. A subset $S \subset X$ is closed in $X$ if and only if $S \cap X_{k}$ is closed in $X_{k}$ for each $k$. Then $X$ is nonmetrizable (it is not first-countable at $(0,0)$ ) and a function $f: X \rightarrow \mathbf{R}$ is continuous if and only if $\left.f\right|_{X}: X_{k} \rightarrow \mathbf{R}$ is continuous for each $k \geqslant 1$. For $k \geqslant 1$ let $C_{k}=\left\{g \in \mathbf{R}^{X}|g|_{X_{k}}\right.$ is continuous on $\left.X_{k}\right\}$. The proof of Proposition 8 can be modified to show that each $C_{k}$ is an $F_{\sigma \delta}$ subset of $\mathbf{R}^{X}$. Hence so is $C_{\pi}(X)=\bigcap_{k=1}^{\infty} C_{k}$.

Our final examples show the importance of the complete Hausdorff property in Theorems 1,6 and 7. 
10. ExAmple. There is a nondiscrete regular $T_{1}$-space $X$ such that $C_{\pi}(X)$ is a closed (and hence an $F_{\sigma}$ ) subset of $\mathbf{R}^{X}$.

Proof. Let $X$ be any regular $T_{1}$-space such that every continuous, real-valued function on $X$ is constant [E, p. 161]. Then $C_{\pi}(X)$ is a closed subset of $\mathbf{R}^{X}$.

11. EXAMPLE. There is a countable nondiscrete Hausdorff space such that $C_{\pi}(X)$ is a $G_{\delta}$ subset (and hence a $G_{\delta \sigma}$ subset) of $\mathbf{R}^{X}$.

Proof. Let $X$ be a countable, connected, Hausdorff space [W, p. 196]. Then every continuous, real-valued function on $X$ is constant, so that $C_{\pi}(X)$ is a closed subset of the metrizable space $\mathbf{R}^{X}$. Hence $C_{\pi}(X)$ is a $G_{\delta}$ set in $\mathbf{R}^{X}$.

\section{BIBLIOGRAPHY}

[E] R. Engelking, General topology, PWN, Warsaw, 1977.

[dG] J. de Groot, Subcompactness and the Baire category theory, Indag. Math. 25 (1963), 761-767.

[LMP] D. Lutzer, J. van Mill and R. Pol, Descriptive complexity of function spaces, Trans. Amer. Math. Soc. (to appear).

[LM] D. Lutzer and R. McCoy, Category in function spaces. I, Pacific J. Math. 90 (1980), 145-168.

[W] S. Willard, General topology, Addison-Wesley, Reading, Mass., 1970.

Department of Mathematics and Statistics, Miami University, Oxford, Ohio 45056 (Current address of T. Grilliot and D. Lutzer)

Department of Mathematics, Vrije Universiteit, Amsterdam, The Netherlands (Current address of Jan van Mill)

Department of Mathematics, University of Amsterdam, Amsterdam, The Netherlands (Current address of J. Dijkstra) 